\title{
A Generalized Ky Fan Minimax Inequality on Finite-Dimensional Spaces
}

\author{
Marco Castellani $^{1} \cdot$ Massimiliano Giuli $^{1}$
}

Received: 19 January 2021 / Accepted: 27 June 2021 / Published online: 15 July 2021

(C) The Author(s) 2021

\begin{abstract}
An existence result for a generalized inequality over a possible unbounded domain in a finite-dimensional space is established. The proof technique allows to avoid any monotonicity assumption. We adapt a weak coercivity condition introduced in Castellani and Giuli (J Glob Optim 75:163-176, 2019) for a generalized game which extends an older one proposed by Konnov and Dyabilkin (J Glob Optim 49:575-577, 2011) for equilibrium problems. Our main result encompasses and generalizes several existence results for equilibrium, quasiequilibrium and fixed-point problems.
\end{abstract}

Keywords Quasiequilibrium problem · Ky Fan minimax inequality · Fixed point · Coercivity condition

Mathematics Subject Classification 47J20 - 49J35 - 49J40 - 90C30

\section{Introduction}

Ky Fan established his famous minimax inequality result which concerns the existence of solutions for an inequality of minimax type that nowadays is called in the literature "equilibrium problem" [13]. Such a model has gained a lot of interest in the last decades because it has been used in different contexts as economics, engineering, physics, chemistry and so on (see [5] for a recent comprehensive summary covering the main results concerning the existence of equilibria and the solution methods for finding them).

A more general setting where the constraint set depends on the current analyzed point was studied for the first time in the context of impulse control problem [2], and it has been subsequently used by several authors for describing a lot of problems

$凶$ Massimiliano Giuli

massimiliano.giuli@univaq.it

1 Department of Information Engineering, Computer Science and Mathematics, Via Vetoio, Coppito, AQ 67100, Italy 
that arise in different fields: equilibrium problems in mechanics, Nash equilibrium problems, equilibria in economics, network equilibrium problems and so on.

Joly and Mosco [15] described a unifying framework for studying both the problems at the same time, and they established existence results by applying the Kakutani fixedpoint theorem to a suitable defined selection set-valued map.

The aim of this paper is to establish a result concerning existence of solutions for such a class of variational problems defined on a finite-dimensional space. Our approach is based on a Michael selection result for lower semicontinuous set-valued maps in the spirit of [9], combined with a weak coercivity condition introduced in [6] and without requiring any assumption of generalized monotonicity. Our result subsumes and extends several similar results which can be obtained by means of suitable choices of the involved functions.

\section{The Problem and the Notations}

A great number of mathematical equilibrium models (fixed points, Nash games, variational inequalities, complementarity problems, optimization problems and so on), which are apparently different, have a common structure that leads to a unified format: the Ky Fan inequality also called equilibrium problem which asks to

$$
\text { find } x \in C \text { such that } f(x, y) \geq 0 \text { for all } y \in C \text {, }
$$

where $C \subseteq \mathbb{R}^{n}$ is a nonempty set and $f: C \times C \rightarrow \mathbb{R}$ is a bifunction.

In the equilibrium problems, the constraint set is fixed and hence the model cannot be used in many cases where the constraints depend on the current analyzed point. This more general setting, commonly called quasiequilibrium problem, reads

$$
\text { find } x \in K(x) \text { such that } f(x, y) \geq 0 \text { for all } y \in K(x) \text {, }
$$

where the constraint $K: C \rightrightarrows C$ is a set-valued map.

Joly and Mosco [15] studied a class of variational problems involving an extended valued bifunction $\varphi: C \times C \rightarrow(-\infty,+\infty]$ which captures the nature of the constraint. This problem asks to

$$
\text { find } x \in C \text { such that } f(x, y)+\varphi(x, y) \geq \varphi(x, x) \text {, for all } y \in C \text {, }
$$

where the domain of $\varphi(x, \cdot)$

$$
D_{\varphi}(x)=\{y \in C: \varphi(x, y)<+\infty\}
$$

is assumed to be nonempty for every $x \in C$. Problem (3) covers problem (1) choosing $\varphi \equiv 0$. Moreover, problem (2) with $K$ nonempty-valued can be obtained as particular case if $\varphi(x, y)=\delta(y, K(x))$, where $\delta$ is the indicator function defined as follows:

$$
\delta(x, A)= \begin{cases}0 & \text { if } x \in A, \\ +\infty & \text { if } x \notin A,\end{cases}
$$


for all $x \in \mathbb{R}^{n}$ and $A \subseteq \mathbb{R}^{n}$. Furthermore, if $f \equiv 0$ and $\varphi(x, y)=\delta(y, K(x))$, problem (3) consists in finding the fixed points of the set-valued map $K$.

The aim of this paper is to establish an existence result for problem (3) without requiring the compactness of $C$ and any assumptions of generalized monotonicity on $f$ as done in [15] and [14]. We conclude this section reviewing the main definitions and notations used in the sequel.

Given any function $\psi: \mathbb{R}^{n} \rightarrow(-\infty,+\infty]$, the effective domain of $\psi$ is defined by dom $\psi=\left\{x \in \mathbb{R}^{n}: \psi(x)<+\infty\right\}$. We say that $\psi$ is a proper function if dom $\psi$ is nonempty. A proper function $\psi$ is said

- to have the sign preserving property if the following implication holds

$$
\psi(x)=0 \text { and } \psi(y)<0 \Rightarrow \psi(t x+(1-t) y)<0, \quad \forall t \in(0,1)
$$

- semistrictly quasiconvex if for all $x, y \in \operatorname{dom} \psi$ such that $\psi(x) \neq \psi(y)$

$$
\psi(t x+(1-t) y)<\max \{\psi(x), \psi(y)\}, \quad \forall t \in(0,1)
$$

- quasiconvex if for every $x, y \in \operatorname{dom} \psi$

$$
\psi(t x+(1-t) y) \leq \max \{\psi(x), \psi(y)\}, \quad \forall t \in(0,1) .
$$

The sign preserving property was introduced and named in [4], and it is clearly satisfied if $\psi$ is semistrictly quasiconvex. From the geometrical point of view, the quasiconvexity of $\psi$ coincides with the convexity of the sublevel sets

$$
\{x \in C: \psi(x) \leq \alpha\} \text { and }\{x \in C: \psi(x)<\alpha\} \text {, }
$$

for all $\alpha \in \mathbb{R}$. Simple examples show there is no relationship between quasiconvexity and semistrict quasiconvexity. However, under lower semicontinuity, semistrict quasiconvexity implies quasiconvexity.

Let $\Psi: X \rightrightarrows Y$ be a set-valued map with $X$ and $Y$ two topological spaces. The domain of $\Psi$ is $\operatorname{dom} \Psi=\{x \in X: \Psi(x) \neq \emptyset\}$. The map $\Psi$ is said to be

- lower semicontinuous at $x$ if for each open set $\Omega$ such that $\Psi(x) \cap \Omega \neq \emptyset$ there exists a neighborhood $U_{x} \in \mathcal{N}_{x}$ such that $\Psi\left(x^{\prime}\right) \cap \Omega \neq \emptyset$ for every $x^{\prime} \in U_{x}$;

- upper semicontinuous at $x$ if for each open set $\Omega$ such that $\Psi(x) \subseteq \Omega$ there exists a neighborhood $U_{x} \in \mathcal{N}_{x}$ such that $\Psi\left(x^{\prime}\right) \subseteq \Omega$ for every $x^{\prime} \in U_{x}$.

The map $\Psi$ is said to be lower (upper) semicontinuous if it is lower (upper) semicontinuous at each $x \in X$. The domain of a lower semicontinuous set-valued map is open.

A fixed point of a set-valued map $\Psi: X \rightrightarrows X$ is a point $x \in X$ satisfying $x \in \Psi(x)$. The set of the fixed points of $\Psi$ is denoted by fix $\Psi$. We recall that under the assumption of compactness of $C$, the upper semicontinuity and the closed valuedness of $\Psi$ are equivalent to the closedness of the graph of $\Psi$ which implies the closedness of fix $\Psi$, while the converse is not necessarily true. A selection of $\Psi$ is a function $\psi: X \rightarrow Y$ that satisfies $\psi(x) \in \Psi(x)$ for each $x \in X$. 
Given a subset $A \subseteq \mathbb{R}^{n}, \operatorname{cl} A$ denotes its topological closure and co $A$ its convex hull. If $\Psi$ is a set-valued map, co $\Psi$ is the set-valued map defined by $(\operatorname{co} \Psi)(x)=$ co $\Psi(x)$. We recall that convex hull of a lower semicontinuous map is itself lower semicontinuous. Lastly, the open ball centered at the origin with radius $r$ will be denoted by $B_{r}$.

\section{The Main Result}

Associated with problem (3), we define the set-valued map $F: C \rightrightarrows C$ as

$$
\begin{aligned}
F(x) & =\{y \in C: f(x, y)+\varphi(x, y)<\varphi(x, x)\} \\
& = \begin{cases}\{y \in C: \psi(x, y)<0\} & \text { if } x \in \text { fix } D_{\varphi}, \\
D_{\varphi}(x) & \text { if } x \notin \text { fix } D_{\varphi},\end{cases}
\end{aligned}
$$

where $\psi:$ fix $D_{\varphi} \times C \rightarrow(-\infty,+\infty]$ is defined by $\psi(x, y)=f(x, y)+\varphi(x, y)-$ $\varphi(x, x)$. Clearly, if $x$ solves (3), then $x$ is a maximal element of $F$, i.e., $F(x)=\emptyset$, while the reverse implication holds since we have assumed that $D_{\varphi}(x) \neq \emptyset$ for every $x$. The main result of the paper is the following:

Theorem 1 Let $C \subseteq \mathbb{R}^{n}$ be a nonempty closed convex set. Assume that $f(x, x)=0$ for all $x \in$ fix $D_{\varphi}$ and

(i) fix co $F=\emptyset$,

(ii) fix $D_{\varphi}$ is closed,

(iii) $D_{\varphi}$ is lower semicontinuous and convex-valued,

(iv) co $F$ is lower semicontinuous on fix $D_{\varphi}$,

(v) $\psi(x, \cdot)$ has the sign preserving property for each $x \in$ fix $D_{\varphi}$.

If there exist a quasiconvex continuous function $\mu: \mathbb{R}^{n} \rightarrow \mathbb{R}$ and $r \in \mathbb{R}$ such that

(a) $C \cap W_{r} \neq \emptyset$ is bounded and $D_{\varphi}(x) \cap L_{r} \neq \emptyset$ for each $x \in C \cap W_{r}$ where $W_{r}=\left\{x \in \mathbb{R}^{n}: \mu(x) \leq r\right\}$ and $L_{r}=\left\{x \in \mathbb{R}^{n}: \mu(x)<r\right\}$,

(b) for each $x \in$ fix $D_{\varphi}$ with $\mu(x)=r$ there is a point $y \in D_{\varphi}(x)$ with $\mu(y)<r$ such that $\psi(x, y) \leq 0$,

then problem (3) has a solution.

Proof Let $C_{r}=C \cap W_{r} \neq \varnothing$. Since $L_{r}$ is an open set, the map $F^{r}: C_{r} \rightrightarrows C_{r}$ defined as $F^{r}(x)=\operatorname{co} F(x) \cap L_{r}$ is lower semicontinuous on fix $D_{\varphi} \cap C_{r}$ and the map $D_{\varphi}^{r}: C_{r} \rightrightarrows C_{r}$ defined as $D_{\varphi}^{r}(x)=D_{\varphi}(x) \cap L_{r}$ is lower semicontinuous on $C_{r}$.

We show that $F^{r}$ is lower semicontinuous on the whole set $C_{r}$. The set-valued map $F^{r}$ is a submap of $D_{\varphi}^{r}$, that is, $F^{r}(x) \subseteq D_{\varphi}^{r}(x)$ for all $x \in C_{r}$. Moreover,

$$
F^{r}(x)=D_{\varphi}^{r}(x), \quad \forall x \in C_{r} \backslash \text { fix } D_{\varphi} .
$$

Since $D_{\varphi}^{r}$ is lower semicontinuous on the set $C_{r} \backslash$ fix $D_{\varphi}$ which is open in $C_{r}$, for proving the lower semicontinuity of $F^{r}$ it is enough to show its lower semicontinuity at any $x \in$ fix $D_{\varphi} \cap C_{r}$. Take an open set $\Omega \subseteq \mathbb{R}^{n}$ such that $F^{r}(x) \cap \Omega \neq \emptyset$. From the 
lower semicontinuity at $x$ of $F^{r}$ and $D_{\varphi}^{r}$, there are two neighborhoods $U_{x}^{1}, U_{x}^{2} \in \mathcal{N}_{x}$ such that

$$
\begin{aligned}
& F^{r}\left(x^{\prime}\right) \cap \Omega \neq \emptyset, \quad \forall x^{\prime} \in U_{x}^{1} \cap \text { fix } D_{\varphi} \cap C_{r} \\
& D_{\varphi}^{r}\left(x^{\prime}\right) \cap \Omega \neq \emptyset, \quad \forall x^{\prime} \in U_{x}^{2} \cap C_{r} .
\end{aligned}
$$

Hence, choosing $U_{x}=U_{x}^{1} \cap U_{x}^{2}$ we have $F^{r}\left(x^{\prime}\right) \cap \Omega \neq \emptyset$ for every $x^{\prime} \in U_{x} \cap C_{r}$.

By contradiction, assume that $F^{r}(x) \neq \varnothing$ for all $x \in C_{r}$. One of the famous Michael's selection results affirms that every lower semicontinuous set-valued map from a metric space to $\mathbb{R}^{n}$ with nonempty convex values admits a continuous selection [18, Theorem 3.1"' (b)]. Let $g: C_{r} \rightarrow C_{r}$ be a continuous selection of $F^{r}$. The Brouwer fixed-point theorem provides the existence of a fixed point of $g$ which in turn is a fixed point of $F^{r}$ and it contradicts (i). Then, there exists $x \in C_{r}$ such that $F^{r}(x)=\emptyset$. From (4) and (a), the maximal point $x$ belongs to fix $D_{\varphi}$ and

$$
\left\{y \in C \cap L_{r}: \psi(x, y)<0\right\}=\emptyset .
$$

We claim that $x$ is a solution of (3). By contradiction, let $z \in C$ with $\mu(z) \geq r$ such that $z \in F(x)$. We distinguish two cases.

Case $\mu(x)<r$. Since $C$ is a convex set and $\mu$ is a continuous function, $z_{t}=x+t(z-$ $x) \in C \cap L_{r}$, for $t>0$ small enough. Furthermore, $x \in$ fix $D_{\varphi}$ then $\psi(x, x)=0$ and $\psi(x, z)<0$, and (v) implies $\psi\left(x, z_{t}\right)<0$.

Case $\mu(x)=r$. Assumption (b) implies there exists $y \in C \cap L_{r}$ such that $\psi(x, y) \leq 0$. From (5), we have $\psi(x, y)=0$ and $\psi(x, z)<0$; hence $z_{t}=y+t(z-$ $y) \in C \cap L_{r}$ for $t>0$ sufficiently small and (v) implies $\psi\left(x, z_{t}\right)<0$.

In both cases, $z_{t} \in F^{r}(x)$ which contradicts (5).

Remark 1 Let us focus on the case where $C$ is compact. Choosing $\mu=\|\cdot\|$ and $r$ sufficiently large, conditions (a) and (b) are trivially verified. Moreover, as can be deduced from the proof, the requirement of $f(x, x)=0$ for all $x \in$ fix $D_{\varphi}$ and the sign preserving property of the function $\psi(x, \cdot)$ are not necessary, and Theorem 1 includes [9, Theorem 2.1]. Anyway, we stress the fact that $f(x, x) \geq 0$ for all $x \in$ fix $D_{\varphi}$ is a direct consequence of (i).

Let us dwell for a moment on the necessity of the finite dimensionality of the spaces. This assumption plays an important role in establishing both the existence of the selection $g$ of $F^{r}$ and the existence of a fixed point of $g$. Indeed, Theorem 3.1"' (b) in [18] does not hold if the finite dimensionality is omitted (see Example 6.3 in [18]). In order to overcome this difficulty, a possible alternative is to require the values of $D_{\varphi}$ being in a particular family of convex sets (see, for instance, [7]). Anyway, even if the continuous selection $g$ exists, the Brouwer fixed-point theorem could fail due to the lack of compactness of $C_{r}$. The following example is inspired by the construction due to Kakutani in [16].

Example 1 Let $\left\{y_{k}\right\}$ with $k \in \mathbb{Z}$ be a orthogonal normalized system of a separable infinite-dimensional Hilbert space $H$ and $U: H \rightarrow H$ be the unitary transformation 
defined by $U\left(y_{k}\right)=y_{k+1}$ for each $k \in \mathbb{Z}$. Take $C=\{x \in H:\|x\| \leq 1\}$. The function $K$ defined by $K(x)=(1-\|x\|) y_{0} / 2+U(x)$ is a homeomorphism of $C$ onto itself which has no fixed point [16, Theorem 1]. For any problem (3) with $\varphi(x, y)=\delta(y, K(x))$, we have $D_{\varphi}(x)=K(x)=F(x)$ since $D_{\varphi}(x)$ has not fixed points. Hence, all assumptions (i)-(v) of Theorem 1 are trivially satisfied. Moreover, the coercivity condition is also verified by choosing $r=2$ and $\mu=\|\cdot\|$. Anyway, problem (3) has not solution since $F(x) \neq \emptyset$ for each $x \in C$.

The variational problem (3) was introduced in [15] where some existence results were proved. Such results are based on the fact that the solutions of (3) coincide with the fixed points of the so-called variational selection map $S: C \rightrightarrows C$ defined by

$$
S(x)=\{z \in C: f(z, y)+\varphi(x, y) \geq \varphi(x, z), \forall y \in C\}
$$

and the existence of a fixed point of $S$ descends from the Kakutani's fixed-point theorem. In order to apply the Kakutani theorem, the authors required the strong assumption of convexity of $f(z, \cdot)+\varphi(x, \cdot)$ and the monotonicity of $f$. More recently, based on the same approach and on recession notions which proved to be very useful in the study of noncoercive minimization problems, Florez-Bazán [14] extended the results in [15] when the space is a reflexive Banach space. Anyway, in both papers the monotonicity of $f$ is a crucial tool for proving the existence of solutions of an auxiliary Minty-type variational problem.

When the set of fix $D_{\varphi}$ is countable the coercivity condition can be considerably simplified.

Theorem 2 Let $C \subseteq \mathbb{R}^{n}$ be a nonempty closed convex set. Assume that $f(x, x)=0$ for all $x \in$ fix $D_{\varphi}$ and all assumptions (i)-(v) in Theorem 1 hold. If fix $D_{\varphi}$ is countable, then condition (a) is sufficient for the existence of solutions for problem (3).

Proof We will show that if (a) holds for a suitable $r \in \mathbb{R}$, then there exists $R>r$ such that (a) is fulfilled for each $s \in[r, R)$. Once obtained, since fix $D_{\varphi}$ is countable, there exists a suitable $s \in[r, R)$ such that $\mu(x) \neq s$ for all $x \in$ fix $D_{\varphi}$ (and hence also condition (b) is fulfilled).

The first step consists in showing the existence of $R>r$ such that $C \cap W_{R}$ is bounded: The nonemptiness and the boundedness of $C \cap W_{s}$ for each $s \in(r, R)$ follow from the fact that $W_{s} \subseteq W_{R}$ for all $s<R$ and $C \cap W_{r} \neq \emptyset$. By assumption, there exists $\rho>0$ such that $C \cap W_{r} \subset B_{\rho}$ and suppose, by contradiction, that $C \cap W_{s}$ are unbounded for all $s>r$. For each $r_{k}=r+1 / k$ with $k \in \mathbb{N}$, there exists $x_{k} \in C \cap W_{r_{k}}$ with $\left\|x_{k}\right\|>\rho$. Fixed $x \in C \cap W_{r}$, the line segment joining $x$ to $x_{k}$ meets $\operatorname{cl} B_{\rho}$ at a point $z_{k} \in C \cap W_{r_{k}}$ with $\left\|z_{k}\right\|=\rho$. Since the sequence $\left\{z_{k}\right\}$ is bounded, there exists a subsequence $\left\{z_{k_{h}}\right\}$ which converges to a suitable $z \in C$ with $\|z\|=\rho$. Moreover,

$$
\mu(z)=\lim _{h \rightarrow \infty} \mu\left(z_{k_{h}}\right) \leq \lim _{h \rightarrow \infty} r_{k_{h}}=r
$$

and $z \in C \cap W_{r}$ which contradicts the fact $\|z\|=\rho$. 
The next step consists in showing that there exists $R^{\prime}>r$ such that $D_{\varphi}(x) \cap L_{s} \neq \varnothing$ for each $s \in\left(r, R^{\prime}\right)$ and each $x \in C \cap W_{s}$. Once again, assume by contradiction that there exist $\left\{r_{k}\right\} \downarrow r$ and $\left\{x_{k}\right\}$ such that $x_{k} \in C \cap W_{r_{k}}$ and $D_{\varphi}\left(x_{k}\right) \cap L_{r_{k}}=\emptyset$ for each $k$. Since the sequence $\left\{x_{k}\right\}$ is afterward inside $C \cap W_{R}$ which is bounded, it follows that there exist $x \in C$ and a subsequence $\left\{x_{k_{h}}\right\}$ which converges to $x$. Moreover, thanks to the continuity of $\mu$,

$$
\mu(x)=\lim _{h \rightarrow \infty} \mu\left(x_{k_{h}}\right) \leq \lim _{h \rightarrow \infty} r_{k_{h}}=r
$$

which implies $x \in C \cap W_{r}$. By assumption, $x$ belongs to the domain of the lower semicontinuous set-valued map $D_{\varphi} \cap L_{r}$ that is open: This fact contradicts $D_{\varphi}\left(x_{k}\right) \cap$ $L_{r} \subset D_{\varphi}\left(x_{k}\right) \cap L_{r_{k}}=\emptyset$ for all $k$.

\section{Particular Cases}

This section deals with the application of Theorem 1 when $f$ and $\varphi$ are properly chosen.

\subsection{The Case $\varphi \equiv 0$}

In this case, problem (3) collapses to the Ky Fan inequality (1) with

$$
F(x)=\{y \in C: f(x, y)<0\} \text { and } D_{\varphi}(x)=C,
$$

for each $x \in C$. Assumptions (ii) and (iii) of Theorem 1 hold trivially. Assumption (v) reads: $f(x, \cdot)$ has the sign preserving property for each $x \in C$. Notice that $x \in \operatorname{co} F(x)$ means that there is a finite subset $A=\left\{y_{1}, \ldots, y_{n}\right\}$ of $C$ such that $f\left(x, y_{i}\right)<0$ and $x \in \operatorname{co} A$. Hence, assumption (i) may be reformulated as follows:

(i') for any finite subset $A$ of $C$ and any $x \in \operatorname{co} A$ there exists $a \in A$ such that $f(x, a) \geq 0$.

We recall that a bifunction $g$ is said to be properly quasimonotone [3] if for every finite set $A$ of $C$ and for every $y \in \operatorname{co} A$ the following inequality is satisfied

$$
\min _{a \in A} g(a, y) \leq 0
$$

Hence, assumption (i) is equivalent to affirm that $g(x, y)=-f(y, x)$ is properly quasimonotone.

Remark 2 Condition ( $\left.\mathrm{i}^{\prime}\right)$ is guaranteed by the convexity of the level set $\{y \in C$ : $f(x, y)<0\}$ (which holds if $f(x, \cdot)$ is quasiconvex) and $f(x, x) \geq 0$ for all $x \in C$.

In [19], it is shown that assumption ( $\left.\mathrm{i}^{\prime}\right)$, together with the closedness of the set $\{x \in C$ : $f(x, y) \geq 0\}$, implies the existence of solutions of the Ky Fan inequality. Anyway, the assumption of closedness of the superlevel set is equivalent to require that $F$ has open 
lower sections which is sufficient for the lower semicontinuity of $F$ and, in turn, it implies the lower semicontinuity of co $F$. Therefore, Theorem 1 contains Theorem 2.3 in [19] as a special case when $C$ is compact while being more general as the following example shows.

Example 2 Consider the Ky Fan inequality associated with $C=[0,1]$ and

$$
f(x, y)= \begin{cases}-1 & \text { if } 0<y=x^{2}<1 \\ 0 & \text { otherwise }\end{cases}
$$

Assumption (i) is trivially satisfied, $\{x \in C: f(x, y) \geq 0\}=[0, \sqrt{y}) \cup(\sqrt{y}, 1]$ is not closed for all $y \in(0,1)$ and

$$
F(x)= \begin{cases}\left\{x^{2}\right\} & \text { if } x \in(0,1) \\ \varnothing & \text { if } x \in\{0,1\}\end{cases}
$$

is lower semicontinuous: Then, Theorem 1 is applicable. The points $x=0$ and $x=1$ are two equilibria.

Notice that a sufficient condition for the lower semicontinuity of $F$ is given by the inequality

$$
\limsup _{x^{\prime} \rightarrow x} \inf _{y^{\prime} \rightarrow y} f\left(x^{\prime}, y^{\prime}\right)=\sup _{U_{y} \in \mathcal{N}_{y}} \inf _{U_{x} \in \mathcal{N}_{x}} \sup _{x^{\prime} \in U_{x}} \inf _{y^{\prime} \in U_{y}} f\left(x^{\prime}, y^{\prime}\right) \leq f(x, y)
$$

for all $(x, y)$. Indeed, let $y \in F(x) \cap \Omega$ be fixed. Then, $f(x, y)<0$ and, choosing $U_{y}=\Omega$ there exists $U_{x}$ such that $\inf _{y^{\prime} \in U_{y}} f\left(x^{\prime}, y^{\prime}\right) \leq f(x, y) / 2<0$ for all $x^{\prime} \in$ $U_{x} \cap X$, that is there is $y^{\prime} \in \Omega$ such that $f\left(x^{\prime}, y^{\prime}\right)<0$. This property is clearly weaker than the upper semicontinuity of $f(x, \cdot)$, and it is verified by the bifunction of the previous example.

As a matter of fact, Theorem 2.3 in [19] has been proved requiring the following coercivity condition: For any sequence $\left\{x_{k}\right\} \subseteq C$ satisfying $\left\|x_{k}\right\| \rightarrow+\infty$, with $x_{k} \in B_{k}$ for every $k$, there exists $k_{0}$ such that for all $k>k_{0}$ it holds that $f\left(x_{k}, y\right) \geq 0$ for all $y \in C \backslash B_{k}$. Anyway, this coercivity condition is not comparable with the coercivity condition described in Theorem 1 which can be simplified as the following: There exist a quasiconvex continuous function $\mu: \mathbb{R}^{n} \rightarrow \mathbb{R}$ and $r \in \mathbb{R}$ such that

(a) $C \cap L_{r} \neq \emptyset$ and $C \cap W_{r}$ is bounded,

(b) for each $x \in C$ with $\mu(x)=r$, there is a point $y \in C$ with $\mu(y)<r$ such that $f(x, y) \leq 0$.

Example 3 This example is from [19]. Let $C=\mathbb{R}$ and $f(x, y)=e^{y^{2}}-x$. Both the assumptions of Theorem 2.3 in [19] and the coercivity condition are obviously verified. The solution set is $(-\infty, 1]$. Instead, Theorem 1 is not applicable. Indeed, every nonempty compact convex set $W_{r}$ is a closed bounded interval, say $\left[x_{1}, x_{2}\right]$, with $\mu\left(x_{1}\right)=\mu\left(x_{2}\right)=r$, and $e^{y^{2}}-x_{1}>0$ for all $y \in\left[x_{1}, x_{2}\right]$. 
Example 4 Let $C=[0, \infty)$ and $f(x, y)=(y-x)(y-2 x+2)$. The coercivity condition in [19] does not hold since $f(k, k+1)=3-k<0$ for all $k>3$. Instead, the coercivity condition of Theorem 1 is verified for $\mu=|\cdot|$ and $r=1$ since the only feasible point $x \in C$ such that $\mu(x)=1$ is $x=1$ and $f(1, y)=y(y-1)<0$ for all $y \in[0,1)$. Moreover, $f(x, \cdot)$ is strictly convex and Theorem 1 guarantees the existence of the solutions of this equilibrium problem: The solutions are $x=0$ and $x=2$.

We conclude with a comparison between our coercivity condition and the coercivity condition presented in [17]: There exist a convex function $\mu: \mathbb{R}^{n} \rightarrow \mathbb{R}$ and $r \in \mathbb{R}$ such that

$(\alpha) C \cap W_{r} \neq \emptyset$ is a bounded set,

( $\beta$ ) for any $x \in C$ with $\mu(x)>r$ there is a point $y \in C$ with $\mu(y)<\mu(x)$ such that $f(x, y) \leq 0$.

If this last condition holds for a convex function $\mu$ and a real number $r$ then $C \cap W_{R}$ is nonempty and bounded for each $R>r$ [20, Corollary 8.7.1]. Then, assumptions (a) and (b) are verified by taking the same function $\mu$ and any $R>r$. The reverse implication does not hold as the following example shows.

Example 5 Assumptions (a) and (b) are verified by the Ky Fan inequality problem described in Example 4. On the converse, for any convex function $\mu$ and any $r$ satisfying $(\alpha)$ we have $C \cap W_{r}=\left[a_{r}, b_{r}\right]$ with $0 \leq a_{r} \leq b_{r}$. Fix $x$ large enough such that $x>\max \left\{2, b_{r}\right\}$ and take any $y \in C$ such that $\mu(y)<\mu(x)$. Since $\mu$ is convex, $y<x$ and therefore $f(x, y)>0$. Hence, assumption $(\beta)$ is not verified.

\subsection{The Case $\varphi(x, y)=\delta(y, K(x))$}

In this case, problem (3) collapses to the quasiequilibrium problem (2) with $D_{\varphi}=K$ which is a nonempty-valued map and

$$
F(x)= \begin{cases}K(x) \cap\{y \in C: f(x, y)<0\} & \text { if } x \in \text { fix } K, \\ K(x) & \text { if } x \notin \text { fix } K\end{cases}
$$

for each $x \in C$. Let us start with a result which clarifies assumption (i) of Theorem 1 .

Lemma 1 The nonemptiness of fix co $F$ is equivalent to affirm that for any $x \in C$ and any A finite subset of $K(x)$ such that $x \in \operatorname{co} A$ there holds $x \in K(x)$ and there exists $a \in A$ such that $f(x, a) \geq 0$.

Proof Assume, by contradiction, that there is $x \in \operatorname{co} F(x)$, i.e., there exists a finite subset $A$ of $F(x)$ such that $x \in \operatorname{co} A$. Two cases are possible. If $x \in$ fix $K$, then $A \subseteq F(x)=K(x) \cap\{y \in C: f(x, y)<0\}$, which means $A \subseteq K(x)$ and $f(x, a)<0$ for all $x \in A$. If $x \notin$ fix $K$, then $A \subseteq F(x)=K(x)$ but $x \notin K(x)$. In both cases, we get a contradiction. Now assume that fix co $F=\emptyset$ and fix a point $x \in C$ and a finite subset $A$ of $K(x)$ such that $x \in \operatorname{co} A$. Clearly $x \in K(x)$. If $f(x, a)<0$ for all $a \in A$, then $A$ is a subset of $\{y \in C: f(x, y)<0\}$ and $x$ would be a fixed point of $\operatorname{co} F$. 
Remark 3 Thanks to Lemma 1 , if $K$ is convex-valued assumption ( $\left.i^{\prime}\right)$ is sufficient to guarantee assumption (i) in Theorem 1, else the following example shows that these two conditions are not comparable. Fix $C=[-2,2]$. The bifunction $f(x, y)=y-x$ satisfies (i') but (i) fails if $K(x)=\{ \pm 1\}$; for instance, $x=0 \in \operatorname{co}\{ \pm 1\}$ but does not belong to $K(0)$. Vice versa the bifunction $f(x, y)=\left(y^{2}-1\right)\left(y^{2}-x^{2}\right)$ does not satisfy (i'): Take $x=0$ and $A=\{ \pm 1 / 2\}$ then $f(0, \pm 1 / 2)=-3 / 16<0$. Nevertheless, if

$$
K(x)= \begin{cases}\{-1,1\} & \text { if } x \in[-2,-1) \cup(1,2], \\ \{-1, x, 1\} & \text { if } x \in[-1,1]\end{cases}
$$

assumption (i) holds.

Assumption (ii) of Theorem 1 becomes fix $K$ closed. Assumption (iii) affirms that $K$ is a lower semicontinuous and convex-valued map, and this implies that (i) can be equivalently rewritten as follows:

$\left(i^{\prime \prime}\right)$ for any $x \in$ fix $K$ and any $A$ finite subset of $K(x)$ such that $x \in \operatorname{co} A$ there exists $a \in A$ such that $f(x, a) \geq 0$.

Let us now come to condition (iv) of Theorem 1 which comes down to the lower semicontinuity of the set-valued map

$$
x \mapsto \operatorname{co}(K(x) \cap\{y \in C: f(x, y)<0\})
$$

on fix $K$. If a map is lower semicontinuous, then so is its convex hull. Therefore, combining Remarks 2 and 3 we notice that Theorem 1 includes [11, Lemma 3.1] in which $K$ is lower semicontinuous, $f(x, x)=0$ and $f(x, \cdot)$ is quasiconvex, for each $x \in C$.

Since the intersection of a lower semicontinuous set-valued map with an open graph set-valued map is lower semicontinuous, a sufficient condition for (iv) is the openness of the graph of the map $x \mapsto\{y \in C: f(x, y)<0\}$. Let us focus on the compact case. We recall that if $C$ is compact assumption (v) as well as $f(x, x)=0$ can be removed (Remark 1) and we get the following result.

Corollary 1 Let $C \subseteq \mathbb{R}^{n}$ be a nonempty compact convex set, $f: C \times C \rightarrow \mathbb{R} a$ bifunction, $K: C \rightrightarrows C$ a set-valued map. Assume that ( $\left.i^{\prime \prime}\right)$ holds, fix $K$ is closed, $K$ is lower semicontinuous, convex-valued and the set $\{(x, y) \in$ fix $K \times C: f(x, y) \geq 0\}$ is closed. Then, the quasiequilibrium problem (2) has a solution.

Corollary 1 contains [12, Theorem 2.1] as a special case, but cases for which the last result does not apply can be easily found.

Example 6 The quasiequilibrium problem (2) associated with $C=[0,5], f(x, y)=$ $(x-y)(y-3)$ and

$$
K(x)=\left\{\begin{array}{l}
(2,5) \text { if } x \in[0,2) \\
{[3,4] \text { if } x \in[2,5]}
\end{array}\right.
$$


has a unique solution at $x=4$. The set fix $K=[3,4]$ is closed, $K$ has open lower sections and $f$ is continuous. The sublevel set $\{y \in[0,5]: f(x, y)<0\}$ is not convex for all $x \in(0,5)$ and [12, Theorem 2.1] does not apply. Anyway, the map

$$
\operatorname{co} F(x)=\left\{\begin{array}{l}
(2,5) \text { if } x \in[0,2), \\
{[3,4] \text { if } x \in[2,3) \cup(4,5],} \\
(x, 4] \text { if } x \in[3,4],
\end{array}\right.
$$

has not fixed points, and all the assumptions of Corollary 1 hold.

Corollary 1 is strongly related to Theorem 3.1 in [8] although not equivalent. Actually, the results are not comparable. Example 6 describes a case where all the assumptions of Corollary 1 hold but $\{y \in[0,5]: f(x, y)<0\}$ is not convex for all $x \in$ fix $K=[3,4]$. Vice versa consider the quasiequilibrium problem (2) associated with $C=[0,2]$, $K(x)=\{2-x\}$ and

$$
f(x, y)= \begin{cases}-1 & \text { if } x \in[0,2) \text { and } y=2 \\ 0 & \text { otherwise }\end{cases}
$$

Then, fix $K=\{1\}$, the assumptions of Theorem 3.1 in [8] are trivially satisfied, but the set $\{(x, y) \in$ fix $K \times C: f(x, y) \geq 0\}=\{1\} \times[0,2)$ is not closed.

More recently, assumption (i') has been used in [10, Theorem 2.3] in the context of a locally convex topological vector space. Nevertheless, their result requires the closedness and the convexity of the values of $K$ which is both upper and lower semicontinuous, and the convexity of the level set $\{x \in C: f(x, y) \geq 0\}$, for each $y \in C$.

Example 7 Take $C=[-2,2], f(x, y)=x^{4}-y^{4}-2 x^{2}+2 y^{2}$ and

$$
K(x)= \begin{cases}{[-3 x-4,-x]} & \text { if } x \in[-2,-1), \\ {[-1,1]} & \text { if } x \in[-1,1], \\ {[-x,-3 x+4]} & \text { if } x \in(1,2]\end{cases}
$$

that is convex-valued and both upper and lower semicontinuous. Even if $f$ is continuous and hence $x \mapsto K(x) \cap\{y \in C: f(x, y)<0\}$ is lower semicontinuous, [10, Theorem 2.3] is not applicable since ( $\left.\mathrm{i}^{\prime}\right)$ is not satisfied at $x=0$ with the choice $A=\{ \pm 2\}$ and $\{x \in C: f(x, y) \geq 0\}$ is not convex for each $y \neq \pm 1$. On the other hand fix $K=[-1,1]$ is closed and ( $\left.\mathrm{i}^{\prime \prime}\right)$ is satisfied. Indeed, $x \in \operatorname{co} A$ with $A \subseteq K(x)$ if and only if $x \in[-1,1]$, i.e., is a fixed point of $K$. Furthermore, if $a_{m}, a_{M} \in A$ are such that $-1 \leq a_{m} \leq a \leq a_{M} \leq 1$ for all $a \in A$, then $x \in\left[a_{m}, a_{M}\right]$ and $f\left(x, a_{m}\right) \geq 0$ if $x \in[-1,0]$ and $f\left(x, a_{M}\right) \geq 0$ if $x \in[0,1]$. Hence, all the assumptions of Theorem 1 hold and the quasiequilibrium problem has a solution: $x=0$.

Let us move to the noncompact case.

Corollary 2 Let $C \subseteq \mathbb{R}^{n}$ be a nonempty closed convex set and suppose that $f$ and $K$ verify the assumptions of Corollary 1. Suppose in addition that $f(x, x)=0$ and $f(x, \cdot)$ has the sign preserving property, for each $x \in$ fix $K$. If there exist a quasiconvex continuous function $\mu: \mathbb{R}^{n} \rightarrow \mathbb{R}$ and $r \in \mathbb{R}$ such that 
(a) $C \cap W_{r} \neq \emptyset$ is bounded and $K(x) \cap L_{r} \neq \emptyset$ for each $x \in C \cap W_{r}$,

(b) for each $x \in$ fix $K$ with $\mu(x)=r$ there is a point $y \in K(x)$ with $\mu(y)<r$ such that $f(x, y) \leq 0$,

then the quasiequilibrium problem (2) has a solution.

Proof It is enough to notice that the sign preserving property of $\psi(x, \cdot)$ is satisfied since $f(x, \cdot)$ has the sign preserving property and $K$ is convex-valued.

Corollary 2 can be viewed as a generalization of [6, Theorem 3]. Indeed, in the cited theorem it is required that the level set $\{y \in C: f(x, y)<0\}$ is convex, for each $x \in$ fix $K$. This assumption, together with $f(x, x)=0$, for each $x \in$ fix $K$ and $K$ convex-valued, guarantees $\left(\mathrm{i}^{\prime \prime}\right)$ (Remarks 2 and 3). There are minor differences, though. The upper semicontinuity of $f(\cdot, y)$ on fix $K$ and the lower semicontinuity of $x \mapsto K(x) \cap\{y \in C: f(x, y)<0\}$ on $\partial_{C}$ fix $K$ assumed in [6, Theorem 3] are here replaced by the closedness of the level set $\{(x, y) \in$ fix $K \times C: f(x, y) \geq 0\}$. Finally, here condition (b) is slightly simplified.

We conclude noticing that Theorem 2 allows us to remove condition (b) in Corollary 2 when the set of fixed point of $K$ is countable.

\subsection{The Case $f \equiv 0$ and $\varphi(x, y)=\delta(y, K(x))$}

In this case, problem (3) collapses to find a fixed point of the set-valued map $K=$ $D_{\varphi}$ which has nonempty values and Theorem 1 is a fixed-point result. First of all, assumption (ii) becomes fix $K$ closed: Anyway, this assumption becomes redundant since fix $K$ not closed implies directly fix $K \neq \emptyset$. Assumption (iii) is equivalent to require that $K$ is a lower semicontinuous convex-valued map. Since

$$
F(x)= \begin{cases}\emptyset & \text { if } x \in \text { fix } K \\ K(x) & \text { if } x \notin \text { fix } K\end{cases}
$$

fix co $F=$ fix $F=\emptyset$. Finally, assumptions (iv) and (v) are trivially satisfied.

Regarding the coercivity condition, assumption (a) becomes $C \cap W_{r} \neq \varnothing$ bounded and $K(x) \cap L_{r} \neq \varnothing$ for each $x \in C \cap W_{r}$, for a suitable quasiconvex continuous function $\mu: \mathbb{R}^{n} \rightarrow \mathbb{R}$ and $r \in \mathbb{R}$. Since $\psi(x, y)=\delta(y, K(x)) \leq 0$ is automatically verified when $y \in K(x)$, then assumption (b) is a direct consequence of (a).

Therefore, Theorem 1 affirms that every lower semicontinuous and nonempty convex-valued map from a closed convex subset of $\mathbb{R}^{n}$ to itself has a fixed point whenever condition (a) is assumed. This result can be viewed as a consequence of the selection result [18, Theorem 3.1'" (b)] and the Brouwer fixed-point theorem.

\section{Conclusions and Further Developments}

In this paper, we have achieved a result concerning the existence of solutions for a class of variational problems defined on a finite-dimensional space. This result subsumes 
and extends some results which can be obtained by means of suitable choices of the involved functions.

Our impression is that problem (3) not only provides a framework for studying different variational problems, but also gives a tool to avoid irregular situations. An example in this direction is based on this simple fact: If we assume

$$
\varphi(x, x)=\inf _{y \in C} \varphi(x, y), \quad \forall x \in D_{\varphi}(x),
$$

then each solution $x \in C$ of the quasiequilibrium problem

$$
\text { find } x \in D_{\varphi}(x) \text { such that } f(x, y) \geq 0 \text { for all } y \in D_{\varphi}(x)
$$

is a solution of (3) since

$$
f(x, y)+\varphi(x, y) \geq f(x, y)+\inf _{y \in C} \varphi(x, y) \geq \inf _{y \in C} \varphi(x, y)=\varphi(x, x) .
$$

How may this fact be worthwhile? Assume that the bifunction of a quasiequilibrium problem can be decomposed as a sum of $g_{1}, g_{2}: C \times C \rightarrow \mathbb{R}$ :

$$
\text { find } x \in K(x) \text { such that } g_{1}(x, y)+g_{2}(x, y) \geq 0 \text { for all } y \in K(x) \text {. }
$$

Consider a quite regular problem: $K$ continuous with nonempty closed convex values, $g_{1}+g_{2}$ upper semicontinuous and nonnegative on the diagonal of $C \times C, g_{1}(x, \cdot)$ convex for each $x \in C$, but there exists $x \in C$ such that $g_{1}(x, \cdot)+g_{2}(x, \cdot)$ is not convex. It is a pity, because the convexity is needed in most existence proofs. By means of the indicator function, let us reformulate (8) in the following way: Find $x \in C$ such that

$$
\begin{aligned}
& {\left[g_{1}(x, y)+g_{2}(x, x)\right]+\left[g_{2}(x, y)+\delta(y, K(x))\right]} \\
& \quad \geq\left[g_{2}(x, x)+\delta(x, K(x))\right], \quad \forall y \in C .
\end{aligned}
$$

Problem (9) is a generalized variational problem (3) with $f(x, y)=g_{1}(x, y)+$ $g_{2}(x, x)$ and $\varphi(x, y)=g_{2}(x, y)+\delta(y, K(x))$. Notice that $D_{\varphi}$ coincides with $K$ and condition (6) becomes

$$
g_{2}(x, x)=\inf _{y \in K(x)} g_{2}(x, y), \quad \forall x \in K(x)
$$

If (10) holds, then we could apply Theorem 6.4.21 in [1] to the quasiequilibrium problem

$$
\text { find } x \in K(x) \text { such that } g_{1}(x, y)+g_{2}(x, x) \geq 0 \text { for all } y \in K(x)
$$

since the objective bifunction is now convex with respect to the second variable. In other words, we have been able to remove the bad part of objective bifunction, at the price of adding a further condition (6). We conclude with a numerical example. 
Example 8 Let $C=[-2,2], g_{1}, g_{2}: C \times C \rightarrow \mathbb{R}$ defined as follows:

$$
g_{1}(x, y)=2 y^{4}-x^{4}, \quad g_{2}(x, y)=\min \left\{1-\sqrt{x^{2}+y^{2}}, 0\right\}
$$

and $K: C \rightrightarrows C$ is given by $K(x)=[-1+|x| / 2,1-|x| / 2]$. The feasible set-valued map is continuous, and $g_{1}$ and $g_{2}$ are continuous with $g_{1}(x, \cdot)$ convex. Unfortunately, $g_{1}(x, \cdot)+g_{2}(x, \cdot)$ is not convex when $|x|>1$. The fixed-point set of $K$ is $[-2 / 3,2 / 3]$. An easy calculation shows that $g_{2}(x, y)=0$ for all $x \in[-2 / 3,2 / 3]$ and $y \in K(x)$ since $x^{2}+y^{2} \leq 1$. Hence, (10) is satisfied and the quasiequilibrium problem has a solution. The unique solution is $x=0$.

This idea may give researchers new insights into choosing appropriate reformulations of equilibrium problems.

Funding Open access funding provided by Universitá degli Studi dell' Aquila within the CRUI-CARE Agreement.

Open Access This article is licensed under a Creative Commons Attribution 4.0 International License, which permits use, sharing, adaptation, distribution and reproduction in any medium or format, as long as you give appropriate credit to the original author(s) and the source, provide a link to the Creative Commons licence, and indicate if changes were made. The images or other third party material in this article are included in the article's Creative Commons licence, unless indicated otherwise in a credit line to the material. If material is not included in the article's Creative Commons licence and your intended use is not permitted by statutory regulation or exceeds the permitted use, you will need to obtain permission directly from the copyright holder. To view a copy of this licence, visit http://creativecommons.org/licenses/by/4.0/.

\section{References}

1. Aubin, J.-P., Ekeland, I.: Applied Nonlinear Analysis. Wiley, New York (1984)

2. Bensoussan, A., Goursat, M., Lions, J.L.: Impulse control and stationary quasi-variational inequalities. C.R. Acad. Sci. Paris Sér. A 276, 1279-1284 (1973)

3. Bianchi, M., Pini, R.: A note on equilibrium problems with properly quasimonotone bifunctions. J. Glob. Optim. 20, 67-76 (2001)

4. Bianchi, M., Pini, R.: A note on stability for parametric equilibrium problems. Oper. Res. Lett. 31, 445-450 (2003)

5. Bigi, G., Castellani, M., Pappalardo, M., Passacantando, M.: Nonlinear Programming Techniques for Equilibria. Springer, Berlin (2019)

6. Castellani, M., Giuli, M.: A coercivity condition for nonmonotone quasiequilibria on finite-dimensional spaces. J. Glob. Optim. 75, 163-176 (2019)

7. Castellani, M., Giuli, M.: Existence of quasiequilibria in metric vector spaces. J. Math. Anal. Appl. 484, 123751 (2020)

8. Castellani, M., Giuli, M., Pappalardo, M.: A Ky Fan minimax inequality for quasiequilibria on finitedimensional spaces. J. Optim. Theory Appl. 179, 53-64 (2018)

9. Castellani, M., Giuli, M., Pappalardo, M.: Existence results for a wide class of equilibrium problems: a general scheme. J. Nonconvex Convex Anal. 21, 1219-1224 (2020)

10. Cotrina, J., Zúñiga, J.: A note on quasi-equilibrium problems. Oper. Res. Lett. 46, 138-140 (2018)

11. Cubiotti, P.: Existence of solutions for lower semicontinuous quasiequilibrium problems. Comput. Math. Appl. 30, 11-22 (1995)

12. Cubiotti, P.: Existence of Nash equilibria for generalized games without upper semicontinuity. Int. J. Game Theory 26, 267-273 (1997)

13. Fan, K.: A minimax inequality and applications. In: Shisha, O. (ed.) Inequalities III, pp. $103-113$. Academic Press, New York (1972) 
14. Flores-Bazán, F.: Existence theorems for generalized noncoercive equilibrium problems: the quasiconvex case. SIAM J. Optim. 11, 675-690 (2000)

15. Joly, J.-L., Mosco, U.: About the existence and the regularity of the solutions of certain quasi-variational inequalities. J. Funct. Anal. 34, 107-137 (1979)

16. Kakutani, S.: Topological properties of the unit sphere of a Hilbert space. Proc. Imp. Acad. Tokyo 19, 269-271 (1943)

17. Konnov, I.V., Dyabilkin, D.A.: Nonmonotone equilibrium problems: coercivity conditions and weak regularization. J. Glob. Optim. 49, 575-577 (2011)

18. Michael, E.: Continuous selections I. Ann. Math. 63, 361-382 (1956)

19. Nasri, M., Sosa, W.: Equilibrium problems and generalized Nash games. Optimization 60, 1161-1170 (2011)

20. Rockafellar, R.T.: Convex Analysis. Princeton University Press, Princeton (1970)

Publisher's Note Springer Nature remains neutral with regard to jurisdictional claims in published maps and institutional affiliations. 